CARATTERIZZAZIONE DEL RISCHIO IN UN AMBIENTE COSTIERO ANTISTANTE UNAREA MINERARIA ED INDUSTRIALE DELLA SARDEGNA (SULCIS-IGLESIENTE) TRAMITE CAMPIONAMENTO A CLUSTER E RICERCA DI CONTAMINANTI SU BIOTI EDULI (PESCI E MOLLUSCHI BIVALVI)

\title{
RISK CHARACTERIZATION OF A COASTAL ENVIRONMENT FACING A MINING AND INDUSTRIAL AREA IN SARDINIA (SULCIS-IGLESIENTE) BY MEANS OF CLUSTER SAMPLING AND CONTAMINANTS RESEARCH ON EDIBLE FISH AND CLAMS
}

Piras P.L. ${ }^{1}$, Mulas G.F. ${ }^{2}$

${ }^{1}$ Servizio Veterinario di Igiene degli Alimenti di Origine Animale dell'Azienda USL 7 di Carbonia; ${ }^{2}$ Servizio Ambiente del Comune di Portoscuso (con finanziamento dell'Assessorato Difesa Ambiente della Regione Sardegna per il Piano di Disinquinamento del Sulcis-Iglesiente)

SUMMARY

KEYWORDS

\begin{abstract}
On the basis of the results obtained from the analysis carried out on various environmental substrates (sediments, sea water and bio-indicators), it can be assumed that there has been a transfer of contaminants from sediments to edible "biota" in the Boi Cerbus lagoon (Sulcis-Iglesiente). It has also been verified that the benthic clams, in particular the Cerastoderma glaucum, is the species mainly affected by heavy metals contamination, in particular Lead, even if the role of Cadmium in the lagoon would need further investigation.
\end{abstract}

food-safety, marine polluting agents, biological indicators.
Sulla base dell'approccio generale alla valutazione e alla gestione dei siti costieri vulnerabili $(1,2)$, le sorgenti di contaminazione si distinguono in puntuali (o locali) e non puntuali (o diffuse). Le sorgenti "puntuali" sono fonti di contaminanti la cui origine è circoscritta e facilmente identificabile. Su tali fonti di contaminazione è concettualmente possibile agire direttamente, sia riducendo le concentrazioni degli inquinanti, sia impedendone la migrazione nei diversi comparti ambientali. Le sorgenti di contaminazione "diffuse" hanno un'origine non circoscritta e rappresentano la sovrapposizione di più eventi e/o attività contaminanti. Le sorgenti vengono poi distinte in primarie e secondarie. La sorgente primaria è rappresentata dall'elemento che è causa di inquinamento (per es. scarichi); quella secondaria è identificata con il comparto ambientale oggetto di contaminazione (per es. i suoli, l'acqua, i sedimenti e, di particolare interesse sotto il profilo della presente indagine, il livello trofico). Nell'ambito della laguna di Boi Cerbus, è senza dubbio riconoscibile come sorgente diffusa il noto fall out determinato dall'area industriale di Portovesme $(3,4) \mathrm{e}$, più in generale, le ricadute persistenti delle vicine aree minerarie dismesse (5). La laguna di Boi Cerbus può inoltre assumere il ruolo di sorgente secondaria, in quanto la sua esposizione ai contaminanti è da mettere in relazione, oltre che all'apporto di particolato aerodisperso (ciminiere), anche ai sedimenti (trasporto solido) e alle acque fluviali (o di ruscellamento) e di falda contaminate, ed è quindi causa di 
ulteriore diffusione dei contaminanti nelle acque marine, nei sedimenti e nei bioti e, in ultima analisi, nell'uomo. L'obiettivo del piano di caratterizzazione svolto nella zona umida di Boi Cerbus è stato quindi quello di evidenziare le interazioni tra le possibili fonti dell'inquinamento del territorio ed i potenziali "bersagli biologici" presenti nelle sue acque di transizione (o "salmastre"), ponendo in evidenza i contaminanti più significativi ed i loro percorsi di migrazione. Porre cioè in relazione le possibili fonti di inquinamento da siti minerari dismessi (dove, lo ricordiamo, venivano prevalentemente estratti Solfuri di $\mathrm{Pb}$ e $\mathrm{Zn}$ ), nonché l'impatto ambientale dell'area industriale ad alto rischio di Portovesme, con lo stato di contaminazione della prospiciente laguna e relativa peschiera, tutt'ora attiva e da valutarsi come possibile sorgente di contaminazione secondaria (6).

Figura 1. Mappa del sito costiero di indagine, dove sono evidenziate le antistanti aree estrattive (sia dismesse che attive) e l'area industriale ad alto rischio (7).

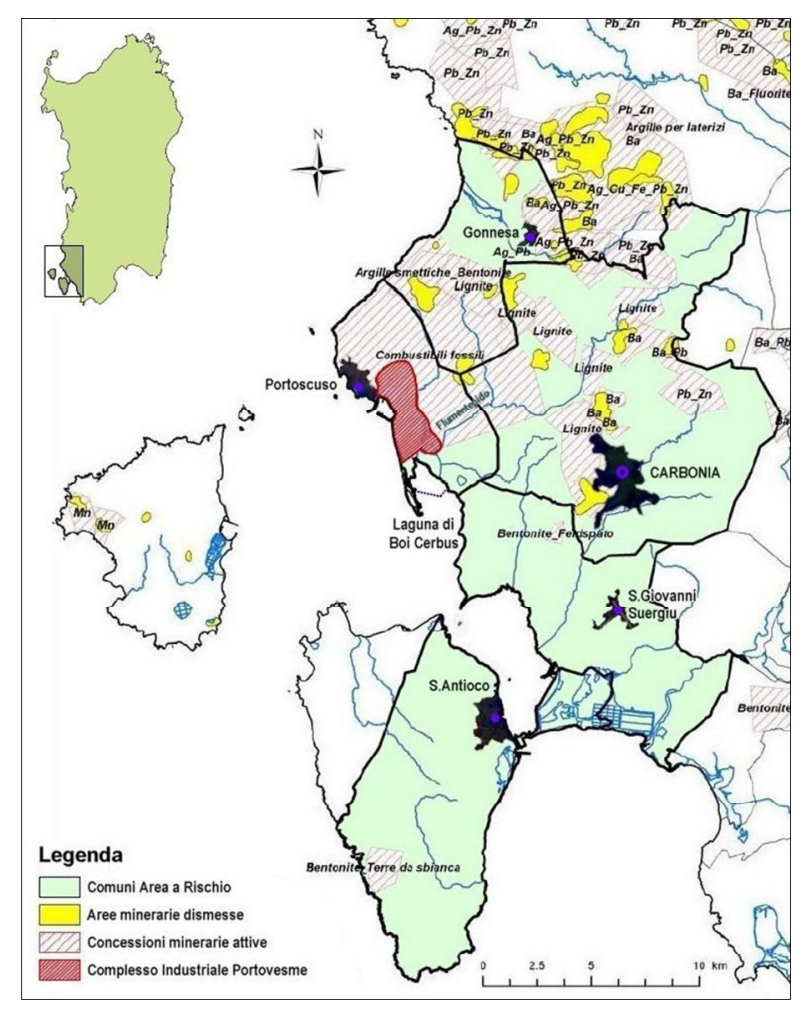

Le lagune infatti, nella loro generalità, assumono la funzione di mero veicolo d'inquinamento per i sistemi biologici in esse compresi e, su un livello trofico superiore, anche per l'uomo che si dovesse alimentare degli organismi acquatici lì pescati o raccolti. L'obiettivo d'indagine è stato raggiunto sviluppando i seguenti aspetti: a) disegnare un profilo fisico-chimico del sito e delle sorgenti di contaminazione, b) rilevare quali siano nello specifico contesto le sostanze inquinanti significative, c) analizzare la distribuzione di tali contaminanti e d) confrontarli con i modelli noti della loro migrazione verso i bersagli biologici finali che, nel nostro caso, sono rappresentati dagli animali acquatici edibili. Relativamente all'uso di animali "sentinella" come validi indicatori biologici, l'area di indagine era infatti già stata monitorata, ma in ambienti di terraferma, tramite il campionamento di miele fresco ed api bottinatrici (8) di cinque stazioni posizionate però nell'area più ampia di tutta la Provincia del Sulcis-Iglesiente. L'area marinocostiera ora indagata (9) ricade invece interamente nel solo territorio comunale di Portoscuso e comprende essenzialmente la parte terminale del bacino idrografico del rio Flumentepido e la laguna di Boi Cerbus, nella quale insiste l'omonima peschiera, dotata del tipico graticciato per la cattura dei pesci eurialini, mugilidi in particolare. Il sito si sviluppa su una superficie di circa $3,5 \mathrm{~km}^{2}$ ed è delimitato, ad Ovest, dalla striscia litorale denominata "Punta S'Aliga" e, a Sud, dal braccio di mare che separa il territorio di Portoscuso dall'isola di Sant'Antioco, mentre a Nord confina col complesso industriale di Portovesme (vedi figura 1 ).

Le attività di analisi eseguite sui campioni delle diverse matrici ambientali (sedimenti, acque salmastre ed organismi "sentinella") della laguna di Boi Cerbus sono state eseguite secondo i Protocolli ICRAM (10), a partire da una rigorosa procedura di posizionamento dei punti di campionamento mediante DGPS (Sistema Differenziale di Posizionamento Satellitare), che l'ARPAS (9) ha validato. Il campionamento delle acque di transizione e, per carotaggio, dei sedimenti della laguna, secondo una maglia di 500 $\mathrm{m}$ di lato (con 18 punti di intersezione), ha consentito di restituire arealmente i risultati ottenuti. La variabilità areale dei parametri chimico-fisici è stata inoltre svolta, nel caso dei sedimenti, per quattro profondità campionate: $0-20$ $\mathrm{cm}, 30-50 \mathrm{~cm}, 100-120 \mathrm{~cm}$ e $180-200 \mathrm{~cm}$. Per il campionamento dei bioti "indicatori ambientali" ci si è avvalsi delle metodiche di pesca e raccolta in uso nella laguna, attraverso l'ausilio della Cooperativa pescatori del Boi Cerbus, titolare della concessione regionale di sfruttamento ittico all'interno dell'omonima peschiera. Tra le specie edibili riportate nello specifico Protocollo ICRAM (10), le attività di pesca hanno riguardato le seguenti specie acquatiche: a) per il gruppo degli organismi nectonici e parzialmente necto-bentonici (in quanto anche limivori di fondo) tipici lagunari, i pesci mugilidi o "Cefali" (con le specie prevalenti Liza ramada e L. aura$t a$, denominate in Italiano rispettivamente: "Ce- 
falo o Calamita o Boltolo" e "Cefalo o Cefalo dorato o Lotregano", più occasionalmente il Chelon labrosus, commercialmente denominato "Cefalo o Bosega", ed il Mugil cephalus, denominato "Cefalo o Volpina", raro in ragione del periodo tardo estivo scelto per il campionamento, coincidente con la sua fase riproduttiva in mare aperto) e b) per il gruppo degli organismi strettamente bentonici, con habitat cioè nel sedimento stesso, un mollusco bivalve (specificamente il Cerastoderma glaucum, commercialmente denominato "Cuore" e relativamente abbondante negli ambienti lagunari della Sardegna).

Tabella 1. Specifiche spazio-temporali per i tre cluster di campionamento dei pesci (procedendo dall'interno della laguna verso il mare aperto) ed indicazione dei punti della cala di pesca, della profondità e delle caratteristiche del pescato.

\begin{tabular}{|c|c|c|c|c|c|}
\hline Punti di prelievo & Coordinate Nord-Est & $\begin{array}{l}\text { prof. } \\
\text { acque }\end{array}$ & $\begin{array}{c}\text { data } \\
\text { prelievo }\end{array}$ & $\begin{array}{l}\text { quantità } \\
\text { pescate }\end{array}$ & $\begin{array}{l}\text { taglia } \\
\text { media }\end{array}$ \\
\hline a) interno graticciato (punti 7-10) & N39 $09^{\prime} 55^{\prime \prime} \mathrm{E} 8^{\circ} 25^{\prime} 04^{\prime \prime}$ & $0,5 \mathrm{~m}$ & $25 / 07 / 2008$ & circa $9 \mathrm{~kg}$ & $25 \mathrm{~cm}$ \\
\hline b) esterno graticciato (punti 15-18) & N39 $08^{\prime} 58^{\prime \prime} \mathrm{E} 8^{\circ} 24^{\prime} 58^{\prime \prime}$ & $0,6 \mathrm{~m}$ & $08 / 09 / 2008$ & circa $10 \mathrm{~kg}$ & $25 \mathrm{~cm}$ \\
\hline c) esterno laguna (loc. Matzaccara) & N39 $08^{\prime} 57^{\prime \prime} \mathrm{E} 8^{\circ} 26^{\prime} 11^{\prime \prime}$ & $0,6 \mathrm{~m}$ & $10 / 09 / 2008$ & circa $11 \mathrm{~kg}$ & $25 \mathrm{~cm}$ \\
\hline
\end{tabular}

Tabella 2: specifiche spazio-temporale per i tre cluster di campionamento dei molluschi bivalvi e dei punti di loro raccolta dal sedimento, oltre che della profondità della colonna d'acqua sovrastante e delle quantità (nette) analizzate.

\begin{tabular}{|l|l|l|l|l|}
\hline \multicolumn{1}{|c|}{ Punti di prelievo } & \multicolumn{1}{|c|}{ Coordinate Nord-Est } & $\begin{array}{c}\text { prof. } \\
\text { acque }\end{array}$ & $\begin{array}{c}\text { data } \\
\text { prelievo }\end{array}$ & $\begin{array}{c}\text { quantità raccolte } \\
\text { (senza guscio) }\end{array}$ \\
\hline a) interno graticciato (punti 5-7) & $\mathrm{N}^{\circ} 9^{\circ} 10^{\prime} 07^{\prime \prime} \mathrm{E} 8^{\circ} 24^{\prime} 53^{\prime \prime}$ & $0,5 \mathrm{~m}$ & $20 / 08 / 2008$ & 185,2 grammi \\
\hline b) esterno graticciato (punti 11-14) & $\mathrm{N}^{\prime} 9^{\circ} 09^{\prime} 51^{\prime \prime} \mathrm{E} 8^{\circ} 25^{\prime} 32^{\prime \prime}$ & $0,5 \mathrm{~m}$ & $29 / 08 / 2008$ & 155,0 grammi \\
\hline c) esterno laguna (loc. Matzaccara) & $\mathrm{N} 39^{\circ} 08^{\prime} 57^{\prime \prime} \mathrm{E} 8^{\circ} 26^{\prime} 11^{\prime \prime}$ & $0,4 \mathrm{~m}$ & $11 / 09 / 2008$ & 256,8 grammi \\
\hline
\end{tabular}

Le determinazioni analitiche eseguite sui sedimenti hanno consentito di tratteggiarne lo stato qualitativo. Non esistendo attualmente limiti legali di riferimento per questo tipo di sedimenti si è ricorso a "valori indicativi" o "di intervento" sito-specifici (background di zona costiera) ricavati dall'ICRAM per l'area confinante di Sant'Antioco attraverso le metodiche analitiche di riferimento reperibili in bibliografia per la "determinazione del contenuto di alcuni elementi chimici nei sedimenti marini” (10). Si passa da valori medi di Piombo pari a $42 \mathrm{mg} / \mathrm{kg}$ s.s. del livello profondo $(180-200 \mathrm{~cm})$ a valori medi di 142 in superficie $(0-20 \mathrm{~cm})$, avendo come valore indicativo di riferimento ICRAM quello di $100 \mathrm{mg} / \mathrm{kg}$ s.s. Analogamente, il Cadmio passa da concentrazioni medie di $2,7 \mathrm{mg} / \mathrm{kg}$ s.s. in profondità, a quelle medie di 20,7 nello strato più superficiale, avendo come valore indicativo di riferimento ICRAM quello di $1,4 \mathrm{mg} / \mathrm{kg}$ s.s. Anche il Mercurio aumenta nelle concentrazioni medie passando dagli strati profondi a quello superficiale, con valori rispettivamente di 0,08 e di $0,4 \mathrm{mg} / \mathrm{kg}$ s.s., $\mathrm{ma}$ in ogni caso significativamente al di sotto del valore indicativo di riferimento ICRAM, fissato in $1,3 \mathrm{mg} / \mathrm{kg}$ s.s. Relativamente agli inquinanti organici, riferendoci al Benzo(a)pirene (generalmente utilizzato come "marcatore" della presenza e degli effetti della generalità degli I.P.A., o "Idrocarburi Poli- ciclici Aromatici" cancerogeni), si e passati da concentrazioni non rilevabili $(<0,001 \mathrm{mg} / \mathrm{kg}$ s.s. $)$ negli strati profondi del sedimento, a valori medi di $0,04 \mathrm{mg} / \mathrm{kg}$ s.s. in quello superficiale, con valore indicativo di riferimento ICRAM pari a $0,76 \mathrm{mg} / \mathrm{kg}$ s.s. Nel caso infine degli "Inquinanti Organici Persistenti”, dosando i diversi congeneri delle diossine e dei PCB diossino-simili (con risultati comunque espressi in Diossinetotali-WHO-TE-upper-bond), si è passato da valori non rilevabili in profondità, a concentrazioni di $0,47 \mathrm{ng} / \mathrm{kg}$ s.s., significativamente al di sotto del valore indicativo di riferimento ICRAM, fissato in $30 \mathrm{ng} / \mathrm{kg}$ s.s. Nel senso della profondità, i campioni che presentano concentrazioni più alte di contaminanti sono quindi i campioni appartenenti al livello più superficiale del carotaggio. A ciò si aggiunga come sia stata rilevata una maggior frequenza di campioni che presentano le concentrazioni più elevate anche procedendo in senso longitudinale, cioè dall'interno del graticciato verso l'esterno dello stesso.

Anche le determinazioni analitiche eseguite sui campioni della colonna d'acqua lagunare hanno consentito di definirne lo stato qualitativo, ricorrendo stavolta a limiti normati (D.Lgs n. 152/2006). Mentre per Benzo(a)pirene, Diossine-totali-WHO-TE-upper-bond e Mercurio si è rimasti sotto i rispettivi limiti di rilevabilità, 
nel caso del Piombo è stato rilevato un valore medio di $1,38 \mu \mathrm{g} / \mathrm{l}$ (limite $10 \mathrm{\mu g} / \mathrm{l}$ ) ed in quello del Cadmio di 0,3 $\mathrm{\mu g} / \mathrm{l}$ (limite $1 \mathrm{\mu g} / \mathrm{l})$, significativamente al di sotto dei valori raccomandati. Infine, le analisi chimico-fisiche eseguite sui campioni di biota hanno consentito di completare la caratterizzazione dell'ambiente, ricorrendo stavolta ai limiti previsti dalla regolamentazione comunitaria che definisce i tenori massimi di alcuni contaminanti nei prodotti alimentari (11).

Sulla base dei risultati ottenuti dalle analisi eseguite sulle diverse matrici ambientali (sedimenti, acque di transizione e bioti eduli) si possono quindi confermare i bersagli individuati dai modelli concettuali noti in letteratura (12).

Tabella 3: risultati analitici sulla parte edibile (muscolo fresco) dei mugilidi.

\begin{tabular}{|c|c|c|c|c|c|c|c|c|c|c|}
\hline \multirow{2}{*}{$\begin{array}{c}\text { Contaminanti } \\
\text { nel biota } \\
\text { (pesce) }\end{array}$} & \multirow{2}{*}{$\begin{array}{l}\text { Limite di riferim. } \\
\text { (muscolo di pesce) }\end{array}$} & \multicolumn{3}{|c|}{$\begin{array}{c}\mathbf{A} \\
\text { interno graticciato } \\
\text { (c/o i punti } 7-10)\end{array}$} & \multicolumn{3}{|c|}{$\begin{array}{c}\text { B } \\
\text { esterno graticciato } \\
\text { (c/o i punti 15-18) }\end{array}$} & \multicolumn{3}{|c|}{$\begin{array}{c}\mathrm{C} \\
\text { esterno laguna } \\
\text { (Matzaccara) }\end{array}$} \\
\hline & & $\begin{array}{c}\text { Pool } \\
1\end{array}$ & $\begin{array}{l}\text { Pool } \\
2\end{array}$ & $\begin{array}{c}\text { Pool } \\
3\end{array}$ & $\begin{array}{c}\text { Pool } \\
4\end{array}$ & $\begin{array}{l}\text { Pool } \\
5\end{array}$ & $\begin{array}{c}\text { Pool } \\
6\end{array}$ & $\begin{array}{c}\text { Pool } \\
7\end{array}$ & $\begin{array}{c}\text { Pool } \\
8\end{array}$ & Pool \\
\hline Piombo $(\mathrm{Pb})$ & $\begin{array}{ll}\mathrm{mg} / \mathrm{kg} & 0,30\end{array}$ & 0,21 & 0,09 & 0,12 & 0,17 & 0,36 & 0,20 & 0,13 & 0,19 & 0,23 \\
\hline Cadmio $(\mathrm{Cd})$ & $\begin{array}{ll}\mathrm{mg} / \mathrm{kg} & 0,05\end{array}$ & 0,01 & 0,01 & 0,01 & 0,01 & 0,01 & 0,01 & 0,01 & 0,01 & 0,01 \\
\hline Mercurio $(\mathrm{Hg})$ & $\begin{array}{ll}\mathrm{mg} / \mathrm{kg} & 0,50\end{array}$ & 0,02 & 0,01 & 0,01 & 0,01 & 0,01 & 0,01 & 0,01 & 0,01 & 0,01 \\
\hline Benzo(a)pirene & $\mu \mathrm{g} / \mathrm{kg} \quad 2,0$ & 0,13 & 0,17 & 0,17 & 0,12 & 0,12 & 0,12 & 0,14 & 0,13 & 0,12 \\
\hline Diossine totali & $\mathrm{pg} / \mathrm{kg} \quad 4,0$ & 0,06 & 0,04 & 0,04 & 0,09 & 0,08 & 0,07 & 0,06 & 0,26 & 0,14 \\
\hline
\end{tabular}

Tabella 4: risultati analitici riferiti alla matrice edibile (polpa) dei bivalvi.

\begin{tabular}{|c|c|c|c|c|c|c|c|c|c|c|}
\hline \multirow{2}{*}{$\begin{array}{l}\text { Contaminanti } \\
\text { nel biota } \\
\text { (bivalve) }\end{array}$} & \multirow{2}{*}{$\begin{array}{l}\text { Limite di riferim. } \\
\text { (su polpa sgusciata) }\end{array}$} & \multicolumn{3}{|c|}{$\begin{array}{c}\mathbf{A} \\
\text { interno graticciato } \\
\text { (c/o i punti 5-7) }\end{array}$} & \multicolumn{3}{|c|}{$\begin{array}{c}\text { B } \\
\text { interno graticciato } \\
\text { (c/o i punti 11-14) }\end{array}$} & \multicolumn{3}{|c|}{$\begin{array}{c}\mathrm{C} \\
\text { esterno laguna } \\
\text { (Matzaccara) }\end{array}$} \\
\hline & & $\begin{array}{c}\text { pool } \\
1\end{array}$ & $\begin{array}{c}\text { pool } \\
2\end{array}$ & $\begin{array}{c}\text { pool } \\
3\end{array}$ & $\begin{array}{c}\text { pool } \\
4\end{array}$ & $\begin{array}{c}\text { pool } \\
5\end{array}$ & $\begin{array}{c}\text { pool } \\
6\end{array}$ & $\begin{array}{c}\text { pool } \\
7\end{array}$ & $\begin{array}{c}\text { pool } \\
8\end{array}$ & $\begin{array}{c}\text { pool } \\
9\end{array}$ \\
\hline Piombo $(\mathrm{Pb})$ & \begin{tabular}{|ll}
$\mathrm{mg} / \mathrm{kg}$ & 1,5 \\
\end{tabular} & 2,62 & 2,24 & 1,81 & 3,71 & 4,74 & 6,05 & 4,06 & 4,14 & 7,83 \\
\hline Cad & $\begin{array}{|ll|}\mathrm{mg} / \mathrm{kg} & 1,0 \\
\end{array}$ & 0 , & 0,20 & & 0, & 0,49 & 61 & 0,39 & 0,40 & 0,71 \\
\hline Mercurio (Hg) & $\begin{array}{|ll|}\mathrm{mg} / \mathrm{kg} & 0,50 \\
\end{array}$ & 0,02 & 0,01 & 0,01 & 0,0 & 0,02 & 0,03 & 0,02 & 0,03 & 0,05 \\
\hline Benzo(a)pirene & $\mu \mathrm{g} / \mathrm{kg} \quad 10,0$ & 0,09 & 0,10 & 0,07 & 0,10 & 0,10 & 0,12 & 0,11 & 0,10 & 0,12 \\
\hline Diossine totali & $\mathrm{pg} / \mathrm{kg} \quad 4,0$ & 0,02 & 0,05 & 0,02 & 0,02 & 0,02 & 0,02 & 0,04 & 0,03 & 0,06 \\
\hline
\end{tabular}

Come è noto, nel caso di sedimenti contaminati da sostanze con proprietà di bioaccumulo, il percorso di esposizione dominante in termini di rischio sanitario è la catena alimentare, ed in particolare l'ingestione di prodotti ittici pescati 0 raccolti da pescatori occasionali (attività ricreativa) o dai pescatori di sussistenza (attività lavorativa). In funzione dell'uso del sito indagato, non possono infatti essere ritenuti significativi i percorsi di contatto diretto con i sedimenti, quali l'ingestione accidentale e il contatto dermico, non svolgendovisi alcuna attività ricreativa (per esempio nuoto, canottaggio, ecc.). Per quel che concerne le modalità di esposizione dei recettori ecologici, si possono prendere in considerazione entrambe le tipologie di esposizione ai sedimenti contaminati: l'esposizione diretta e quella indiretta. La prima risulta dalla possibile ingestione del sedimento stesso (per esempio da parte di pesci limivori come i mugilidi), oppure è quella che si verifica negli organismi che vivono all'interno dei sedimenti, che sono invece esposti soprattutto attraverso "captazione" (uptake) dei contaminanti dall'acqua interstiziale (come avviene nel caso dei molluschi bivalvi che vivono sui sedimenti). L'esposizione indiretta si realizza invece attraverso la catena alimentare, ovvero tramite il consumo di una preda contaminata. Le comunità bentoniche sembrerebbero quindi quelle maggiormente esposte alla contaminazione diretta in ambiente lagunare, vivendo in contatto continuo con il sedimento. Fondamentale nella valutazione del modello di distribuzione dei contaminanti è stata inoltre la valutazione quali-quantitativa del trasporto dei contaminanti stessi presenti nella matrice sedimento, oltre che dell'effetto che tali meccanismi di trasporto possono avere sull'esposizione e sul rischio. Tale valutazione risulta essere particolarmente complessa in quanto deve tener conto dei processi che condizionano il movimento dei sedimenti e dei contaminati in essi presenti. Inoltre tali fenomeni sono variabili nel tempo e condizionano l'andamento temporale della distribuzione dei contaminanti nelle varie matrici ambientali. In generale il movimento dei sedimenti può avere effetti favorenti o non favorenti l'esposizione ed il rischio (per esempio, gli eventi alluvionali o i regimi di corrente possono determinare il tra- 
sporto di sedimenti, sia contaminati che non, i quali si depositano successivamente). Nello specifico della laguna di Boi Cerbus l'afflusso di sedimenti è possibile per la presenza di vari canali e ruscelli di apporto d'acqua dolce, generalmente però di scarsa portata. Risulterebbe pertanto fortemente limitato sia il fenomeno tramite il quale la movimentazione dei sedimenti possa disperdere la contaminazione ambientale, sia il fenomeno c.d. dellisolamento (mediante interramento) di altri sedimenti contaminati o, infine, la ipotetica riduzione delle concentrazioni di inquinanti (mediante diluizione). In questo quadro, di tendenziale ridotta dinamica dei flussi abiotici, si può dunque ragionevolmente ipotizzare che il contributo della movimentazione dei sedimenti determini unicamente un effetto sfavorevole per quanto concerne l'esposizione ed il rischio nell'ambiente lagunare di Boi Cerbus. Tra le ipotetiche cause di movimentazione dei sedimenti all'interno della laguna (che, oltre ai profili di corrente generati dal, pure scarso, apporto di acque dolci superficiali, come si è detto, dovrebbe riconoscere anche il regime di marea interno alla laguna, comunque fortemente influenzato nella sua dinamica dalla presenza del graticciato della peschiera) si dovrebbe aggiungere anche ogni tipo di bio-turbazione. Tale tipo di cause, tuttavia, non è da ritenersi significativamente impattante. L'importanza della bio-turbazione, infatti, come meccanismo di trasporto nelle lagune è principalmente determinato da fattori come: le caratteristiche del corpo idrico (profondità del letto, salinità e temperatura dell'acqua e loro variazioni stagionali), le caratteristiche del sedimento (per esempio, la sua granulometria e la sua composizione) e la tipologia di organismi (sotto il profilo sia della micro- che della macrofauna). Si può quindi al momento affermare che nella laguna di Boi Cerbus la movimentazione dei contaminanti è prevalentemente legata a quella dei suoi sedimenti e che il biota maggiormente esposto come bersaglio ecologico sia, nel nostro caso, rappresentato dai molluschi bivalvi bentonici, in particolare la specie Cerastoderma glaucum. È di tutta evidenza, infine, come il rischio sia soprattutto associato ai metalli pesanti, in particolar modo al Piombo, anche se il ruolo del Cadmio andrebbe ulteriormente esplorato, ricorrendo anche al contributo di altri bioti "sentinella" come ad es. i crostacei (granchi di estuario).

\section{BIBLIOGRAFIA E DOCUMENTI DI RIFERIMENTO (CON URL INTERNET ATTIVI AL 15 APRILE 2010):}

1. Relazione European Environment Agency (settembre 2000) "Stato e pressioni sull'ambiente marino e costiero del Mediterraneo" - Ufficio delle pubblicazioni ufficiali delle Comunità Europee, 44 pp.: http://www.eea.europa.eu/it/publications/me dsea/at_download/file

2. Relazione No 4/2006 dell'European Environment Agency (2006) "Problemi prioritari per l'ambiente mediterraneo" - Ufficio delle pubblicazioni ufficiali delle Comunità Europee, $\quad 90 \quad$ pp.: http://www.eea.europa.eu/it/publications/ee a_report_2006_4/at_download/file

3. Istituto dell'Ambiente del Centro Comune di Ricerca della Commissione Europea (2000) "Valutazione preliminare dello stato di inquinamento dei suoli, delle acque e dei sedimenti marini nella zona ad alto rischio ambientale di Portoscuso" - Definizione di un sistema di controllo permanente della contaminazione ambientale, 107 pp.: http://publications.jrc.ec.europa.eu/repositor y/bitstream/111111111/9056/1/EUR\%20190 45\%20IT.pdf

4. Relazione del CNR per la VIII Commissione Permanente della Camera dei Deputati (giugno 2007) "Relazione sullo stato delle conoscenze in tema di ambiente e salute nelle aree ad alto rischio in Italia" - Consiglio Nazionale delle Ricerche, 135 pp.: http://www.cnr.it/documenti/83_Relazione_a ree_a_rischio.pdf

5. Rapporto ISTISAN 04/28 (dicembre 2004) "Contaminazione ambientale da metalli pesanti connessa con attività mineraria dismessa in Sardegna" - Studio preliminare, Istituto Superiore di Sanità, 72 pp.: http://dspace.iss.it/dspace/bitstream/2198/211831/1/ISSA04_11233117_2004_I_04_28.pdf

6. Regione Autonoma della Sardegna, Assessorato Difesa Ambiente (marzo 2008) "Piano di bonifica delle aree minerarie dismesse del Sulcis-Iglesente" - Direzione Gener. Servizio Tutela dell'Atmosfera e del Territorio, 58 pp.:

http://www.regione.sardegna.it/documenti/1 _19_20080403121548.pdf

7. Regione Autonoma della Sardegna, Direzione Generale ARPAS - Agenzia Regionale Protezione Ambiente Sardegna (giugno 2007) "Determinazione dei valori di fondo 
nelle matrici ambientali dell'area ad alto rischio di Portoscuso" - Progetto operativo, 28 pp.:

http://www.sardegnaambiente.it/documenti/ 21_46_20080214155345.pdf)

8. Massidda M. A. e All., Insect Social Life, XII National Meeting (luglio 2007) "Honey bees as bioindicators of environmental contamination in the industrial and mining area of Sulcis-Iglesiente-Sardinia" - REDIA, XC, 2007: 115-119 http://www.redia.it/images/stories/Abstract_ Redia_2007/19_massidda.pdf

9. Regione Autonoma della Sardegna, Deliberazione n. 4 del Comitato Istituzionale dell'Autorità di Bacino (ottobre 2009) "Caratterizzazione dei corpi idrici della Sardegna" - Relazione Generale ex D.M. n. 131/2008, 83 pp.: http://www.regione.sardegna.it/documenti/1 _73_20091210120722.pdf

10. Ministero Ambiente e Tutela del Territorio, Servizio Difesa del Mare - ICRAM (2001) "Metodologie analitiche di riferimento del programma di monitoraggio per il controllo dell'ambiente marino costiero" - triennio 2001-2003, $114 \quad$ pp.: http://www.apat.gov.it/site/_files/icram/Meto dologieAnalitichediRiferimento_icram.pdf

11. EUR-Lex, l'accesso al diritto dell'Unione Europea (dicembre 2006) "Regolamento (CE) n. 1881/2006 della Commissione del 19 dicembre 2006, che definisce i tenori massimi di alcuni contaminanti nei prodotti alimentari" - G.U.C.E. n. L 364/2006, 26 pp.:

http://eur-

lex.europa.eu/LexUriServ/LexUriServ.do?ur i=CONSLEG:2006R1881:20090701:IT:PDF

12. Rapporto ISTISAN 05/24 (settembre 2005) "Piombo, cadmio, arsenico e mercurio negli alimenti di origine ittica: livelli di presenza e stima delle assunzioni in Italia e nell'Unione Europea" - Workshop sulle problematiche emergenti nel settore dei prodotti ittici, ISS, 104 pp.: http://dspace.iss.it/dspace/bitstream/2198/211745/1/ISSA05_11233117_2005_I_05_24.pdf 